Revista de Psicología de la PUCP. Vol. XV, 2, 1997.

\title{
AUTOESTIMA EN ADOLESCENTES CON BAJO RENDIMIENTO ESCOLAR A TRAVÉS DEL PSICODIAGNÓSTICO DE RORSCHACH
}

\author{
Magally de Garrido-Lecca' \\ Pontificia Universidad Católica del Perí
}

1:] objetivo fue determinar si existen diferencias en la autoestima de adolescentes con bajo rendimiento escolar comparados con los de un rendimiento promedio o superior. Los participantes fueron 60 adolescentes, entre 13 y 16 años, de ambos sexos, con un C.I. promedio o superior al promedio, de los cuales 30 presentaban un bajo rendimiento escolar (grupo de estudio) y 30 un buen rendimiento (grupo contraste). Se administró el WISC$\mathrm{R}$ para determinar el C.I. y el Rorschach según el SC de lixner para evaluat la autoestima. Los resultados mostraron que el Rorschach es adecuado para evaluar la autoestima. Asimismo existe una diferencia significativa en la autoestima de adolescentes con bajo rendimiento como resultado de sus déficits afectivos, en comparación con los de alto rendimiento. Palabras claves: adolescentes, autoestima, bajo rendimiento escolar, WISC-R, Rorschach, Iixner

Self-esteem in adolescents with low academic achievement assessed with the Rorschach Inkblots

The objective of the study was to determine if there were differences in the self-esteem of adolescents with low academic achievement as compared to those with an average or high achievement. The participants were 60 adolescents, aged 13 to 16 , of both sexes and with an average or high IQ. Thirty presented a low school achievement (study group) and 30 had a good achievement (contrast group). The WISC $R$ was administered to determine the IQ and the Rorschach Inkblots, using the CS of lixner, to assess the self-esteem. The results showed that the Rorschach is a good instrument to assess self-esteem. Likewise, there are significant differences in the self-esteem of adolescents with low achievement due to emotional deficits, as compared to those with high achievement.

Keywords: adolescents, self-esteem, low academic achievement, WISC-R, Rorschach, lixner

1. Profesora de la maestría de psicología. Lis licenciada y magister en psicología de la Pontificia Universidad Católica del Perú. Ha realizado investigaciones en niños y adolescentes con bajo rendimiento escolar. Asimismo ha hecho estudios sobre huérfanos sociales, planteando un modelo de afronte a esta problemática. lis miembro de varias sociedades científicasy fundadora de la Sociedad Peruana de Rorschach y su actual vicepresidenta. 

Nos acercamos al siglo XXI y el bajo rendimiento escolar sigue siendo un tema de gran preocupación para la psicología clínica y educativa, no obstante las múltiples investigaciones que se han llevado a cabo. En nuestro medio existe una gran cantidad de alumnos de nivel secundario que presentan un bajo rendimiento escolar, el cual ha tratado de ser explicado, generalmente por los psicólogos educacionales, como el resultado de múltiples factores entre los que podemos mencionar los de tipo biológico, los sociales, e incluso los denominados Problemas Específicos de Aprendizaje.

Durante varios años de nuestra vida profesional, hemos podido apreciar una gran afluencia de niños y adolescentes que llegaban a la consulta privada por presentar bajo rendimiento escolar. En ellos observamos que, curiosamente, se caracterizaban por presentar niveles de inteligencia normal o superior al promedio, y que no presentaban aquellos factores considerados como causales de dicha situación, lo cual nos llevó a pensar que podría existir otro factor de índole psicológico, como podría ser la autoestima por ser un punto básico del proceso de desarrollo y de interferencia en sus aprendizajes escolares.

Teóricamente asumimos que la autoestima es una actitud ${ }^{2}$ hacia un objeto. Es decir partimos del supuesto de que la gente tiene actitudes hacia los objetos y que el si mismo es uno de los objetos hacia el cual se tienen las actitudes (Rosenberg, 1983). La autoestima domina la vida subjetiva del individuo, determinando en gran medida sus pensamientos, sus sentimientos y su conducta. A primera vista parece tratarse de un fenómeno

2. lil término actitud posee alcances bastante amplios, se le emplea para designar hechos, opiniones, valores y orientaciones favorables, o desfavorables, respecto de si mismo 
exclusivamente individual, personal o idiosincrático, sin embargo, el autorretrato del individuo no constituye una obra estrictamente subjetiva, sino es más bien un retrato más o menos claro basado en la información recibida de su experiencia social.

La adolescencia puede ser considerada, como señala Caplan (1983), de muy diferentes maneras y desde muchas perspectivas distintas. Difiere de acuerdo con las épocas, las culturas y los medios sociales. Desde el punto de vista de la psicología se sabe menos acerca de la adolescencia que respecto de la niñez, probablemente debido a la mayor variedad de sus modos de manifestarse. Cualquier intento de síntesis podría resultar incompleto, pues algunos lo encontrarian aceptable, pero para otros será discutible.

Jeammet (1993) hace referencia a las características psicodinámicas de la adolescencia. La pubertad dicen puede ser observada como un factor de excitación que desequilibra la organización psíquica en el lugar. La pubertad es un factor de sexualización de lazos con respecto a objetos internos y de aquellos de la realidad exterior, pero también de ambas instancias. Es una etapa de reactivación de conflictos infantiles y de angustias de separación como de castración, que favorecen la regresión, y la reactivación de fijaciones infantiles.

La adolescencia tiene efectos potencialmente traumáticos, es decir susceptibles de desorganizar las capacidades de funcionamiento del Yo. Debemos subrayar que este efecto de excitación sobre el funcionamiento psíquico inducido por la pubertad no podría reducirse a datos puramente cuantitativos tales como el aumento de la potencia de pulsiones sexuales, sino que es, ante todo, un conjunto de datos más cualitativos, como el desequilibrio entre los diferentes sistemas de organización que rigen el funcionamiento psíquico traído por la pubertad. Estos sistemas de organización son aquellos clásicos de la metapsicología: sistema Inconsciente, Preconsciente, Consciente del primer tópico; el Ello, Yo, el Superyo y el ideal del Yo del segundo tópico (Freud, 1972). 
Evaluación de la Autoestima en adolescentes con bajo rendimiento escolar

La serie de transformaciones orgánicas características de la pubertad, como es el aumento de la estatura y del peso, la mayor sensibilidad en el olfato, el gusto y el tacto, las modificaciones en la estructura de la piel y la aparición de las características sexuales secundarias o del desarrollo del aparato genital, son cambios que tienen efectos en el nivel del comportamiento, y conducen a revaluaciones y a transformaciones en las actitudes, contribuye a modificar la imagen que tiene el individuo de sí mismo y el modo como percibe a los demás. Todos ellos, finalmente, debido a sus efectos sobre el adolescente, pueden también suscitar nuevas reacciones en las personas que lo rodean.

Esta evaluación del encuentro consigo mismo tiene lugar, simultáneamente, en otro nivel del desarrollo intelectual. Como señala Piaget (1971), en esta etapa se da profunda evolución que experimentan las estructuras intelectuales, caracterizada por la aparición de la lógica en las proposiciones, y por el paso del nivel concreto al hipotético-deductivo en los procesos del pensamiento. Esta evolución del orden intelectual contribuye básicamente a darle su carácter de enfrentamiento y cambio a la mentalidad adolescente.

Es una época en la cual, además, el adolescente tiene que afrontar los numerosos cambios que se producen en las actitudes de las personas que lo rodean, en su posición respecto del grupo social y el rol que se le ofrece. Se le otorgan ciertos derechos y facilidades de los que no disfrutaba de niño, pero a cambio de ello se le imponen nuevas demandas y responsabilidades, y pierde algunos privilegios anteriores.

Para ser él mismo, para afirmarse a sí mismo en su nueva posición, tiene que apartarse y diferenciarse de todo lo que tenga relación con su posición anterior; también sus padres y su medio familiar tendrán que responder por esta afirmación, que en un comienzo es de carácter esencialmente opositor.

La mayoría de los adultos son muy poco tolerantes con esas manifestaciones, que parecen implicar una pérdida de prestigio y una declinación de su autoridad. A menudo reaccionan con observaciones irónicas, 
o con medidas coercitivas, que sólo pueden suscitar la agresividad y reforzar la hostilidad de los jóvenes. Suele ser frecuente que los adolescentes se sientan engañados y desilusionados por sus padres, aunque rara vez el "conflicto de generaciones" llega a ser total, ya que imitándolos o identificándose con ellos definen los contornos de su propia personalidad, aunque sus objetos de identificación no pertenezcan exclusiva, ni necesariamente, al dominio de la realidad. Y por medio de personalidades reales o ficticias que pertenecen a las más diversas categotías de seres humanos y a los más diversos niveles, el adolescente se construye una especie de persona ideal.

Es importante resaltar que para salir de la adolescencia y adquirir una identidad propia y a pesar de la rebeldía con su familia y la necesidad de romper parte de los vínculos que lo unen a ella, el adolescente necesita una imagen positiva de los padres y de su relación mutua. Su autoestima está estrechamente relacionada con la estima en que pueda tener a sus padres.

El adolescente se vuelve muy crítico hacia sus padres, como es el caso de los "dobles mensajes", los padres les exigen conductas o actitudes y en la práctica ellos mismos hacen lo contrario.

Al comienzo de la adolescencia se establece una red de operaciones combinatorias que son la culminación de la evolución intelectual y que, al dat coherencia interna al proceso de pensamiento, serán el criterio de un razonamiento riguroso basado en suposiciones e hipóteis, prescindiendo de su verdad y de su realidad material y sin apelar al control por la experiencia y los hechos. Que de ese modo se superpondrá un mundo construido por el proceso de pensamiento al mundo que revelan los sentidos. Sobre las reglas específicas y circunscritas del niño de más edad se constituye una escala de leyes y principios que gobiernan los campos más diversos: el pensamiento formal se aplicará a todos los contextos y todo será objeto de la organización lógica. De manera que alrededor de los 15 años termine la evolución estructural del pensamiento, precisamente en el nivel de desarrollo en el cual los autores de las escalas para los test 
tropezaron con la imposibilidad de hallar evidencias que distingan dos edades sucesivas (Piaget, 1971).

Apenas puede dudarse, como el mismo Piaget lo ha demostrado, que esta evolución de orden intelectual contribuya profundamente a darle su carácter específico a la mentalidad adolescente. La adquisición de la abstracción permite inquirir en los sistemas de representaciones colectivas que ofrece la cultura en la que crece el sujeto, que se entusiasmará gradualmente por ideas, ideales y valores.

El ingreso a la educación secundaria marca cambios en los tipos de enseñanza, paralelamente aparecen como ciertos los primeros signos puberales. Durante la secundaria siente una angustia subjetiva que sigue a un período en que el desarrollo de las potencialidades afectivas, relacionales y sociales se oponen a la necesidad de restringir su campo de interés al aprobado del final de curso.

Como lo demuestra la sucesión de reformas, la enseñanza secundaria busca adaptarse a la heterogeneidad del alumnado, de sus niveles y de sus intereses. A este proyecto se enfrentan, por una parte, las exigencias y desigualdades sociales $y$, por otra parte, los procesos intrapsíquicos que atraviesa todo individuo en esta etapa y en el que la inestabilidad, el tumulto y la intermitencia son dificultades para un desarrollo idealmente armonioso, en el que se podría responder a todos los problemas de formación y del futuro (Marcelli, 1986).

Ha sido en base a estas consideraciones que nos hemos propuesto realizar un estudio cuyo objetivo general es determinar si existen diferencias en el nivel de la autoestima que presentan los adolescentes de 13 a 16 años de edad de ambos sexos, con niveles intelectuales iguales y superiores al normal, que presentan un bajo rendimiento escolar en comparación con los que tienen un rendimiento escolar superior al promedio.

Existen antecedentes de investigaciones realizadas con relación al tema, como es el caso de Theodora Alcock (1975) quien estudió a niños 
inteligentes y con bajo rendimiento escolar, utilizando la técnica de Rorschach, planteando en su investigación la existencia en ellos de una psicopatología en el desarrollo de la personalidad.

Consideramos relevante la ejecución del presente estudio por ser uno de los pocos que se realizan en el área de la psicología clínica-educacional en nuestro medio y por permitir evaluar empíricamente la relación que existe entre lo intelectual y lo afectivo, lo cual ha sido intuido, pero pocas veces comprobado.

\section{Metodología}

El estudio pertenece a un nivel descriptivo de tipo comparativo, por que se trata de estudiar el nivel de la autoestima que presentan los adolescentes de 13 a 16 años de ambos sexos, con bajo rendimiento escolar, comparados con los de rendimiento escolar prómedio.

\section{Participantes}

Los participantes fueron 60 adolescentes de ambos sexos, entre 13 y 16 años y con niveles intelectuales promedio o superior al promedio. Los adolescentes conformaron dos grupos, uno de estudio $(n=30)$ y otro de contraste $(\mathrm{n}=30)$. El grupo de estudio provinó de la consulta privada, siendo el motivo de consulta el bajo rendimiento escolar, pero éste no era resultado de problemas específicos de aprendizaje. El grupo de contraste estuvo conformado por alumnos provenientes de colegios particulares, análogos a los de la muestra de estudio, pero que tenían un buen rendimiento escolar. Ambos grupos fueron obtenidos a través de un procedimiento de muestreo probabilístico aleatorio simple. Para mayores detalles con relación a los participantes referirse a Garrido Lecca (1995). 
Evaluación de la Autoestima en adolescentes con bajo rendimiento escolar

\section{Instrumentos}

\section{El Psicodiagnóstico de Rorschach}

Esta técnica proyectiva permite evaluar la autoestima y la percepción por sí mismo, que se refiere a la vez a la imagen de sí y a lo vivido o experimentado por esta imagen. Engloba aspectos cognitivos y afectivos de la representación de sí.

\section{Los indices de egocentrismo segün Exner}

$\mathrm{El}$ indice ego constituye uno de los recientes aportes a la investigación con el Psicodiagnóstico de Rorschach (Exner, 1992, 1993). También son importantes los aportes de la escuela francesa (Sanglade, 1982; en AdronikofSanglade, 1993), que nos orientan a creer que es un indicador de las sólidas bases narcisísticas del sujeto, y típico de los adolescentes.

Para evaluar la autoestima se calcula el indice ego junto con factores y variables que detallamos a continuación:

1. El Índice se calcula de la siguiente manera: $3 r+(2) / R$, es decir respuestas de reflejo multiplicado por 3 , más respuestas pares (simétricas) divididas por el número de respuestas del protocolo.

2. Fr-rF son las respuestas en que el área de la mancha es reportada como un reflejo o imagen de un espejo, basada en la simetría de la mancha.

3. Kinestésicas son las respuestas $M$ de movimiento humano.

4. FD es un determinante que traduce las perspectivas basadas en las características formales de la mancha (líneas de huida).

5. Vista, traduce el difumado, relieve, profundidad, transparencia.

6. H, son los contenidos humanos; parte de contenidos humanos.

7. Xy es una categoría de contenido para radiografías, An=Anatomía.

8. MOR es una calificación especial atribuida a objetos estropeados, destruidos, deteriorados, o a afectos negativos. 
Es necesario indicar que de estas 8 variables estudiadas sólo (Fr-rF) no pertenece a las utilizadas por Sanglade en 1982 (Andronikof Sanglade, 1993) y que ha sido incluido en el presente estudio independiente de la proporción $(3 r+(2) / R)$, tomando en cuenta lo señalado por Exner (1993), quién indica que cuando aparece una respuesta de Reflejo $(\mathrm{Fr})$ en un protocolo debe ser considerada independientemente, ya que probablemente representa una intensa centración en sí mismo.

La interpretación de dichos factores nos dará elementos para conocer las bases narcisísticas, los índices defensivos de tipo aislamiento, la representación de lo humano, capacidad de empatía, preocupación particular por su propio cuerpo, representaciones negativas de sí mismo, representaciones de destrucción, etc.

\section{La Escala de Inteligencia para Niños de Wechsler - revisada}

La Escala de Inteligencia para Niños de Wechsler - revisada (WISCR) mide la inteligencia en niños entre 6 y 16 años, que fue estandarizada en una muestra de 2200 niños estadounidenses. El manual reporta coeficientes de validez y confiabilidad elevados (Wechsler, 1974).

\section{Procedimiento}

Una vez que fueron seleccionados los participantes que conformarían el grupo de estudio con bajo rendimiento escolar, se procedió a recoger toda la información pertinente del archivo de atención psicológica personal. En cuanto al grupo de contraste, una vez seleccionados se les administró individualmente el WISC-R y el Rorschach.

\section{Resultados}

En primer lugar se analizaron las distribuciones de los puntajes obtenidos, observándose que en la variable respuesta de vista (FV) no existian la suficiente cantidad de datos como para ser analizados cuantitativamente de modo tal que se decidió excluirla del resto de análisis estadístico. 
Evaluación de la Autoestima en adolescentes con bajo rendimiento escolar

También pudimos observar que en otras variables era necesario recodificar los datos de manera que sea factible poder analizarlos, como fue el caso de:

1 Movimiento Humano, (M) se reclasificó en 4 categorías $(0,1,2,3)$.

2. Respuesta de Reflejo, (Fr) Respuestas de Dimensionalidad (FD), Anatomia y radiografía (AN+XY), Afectos negativos con matices depresivos (MOR), Contenido Humano $(\mathrm{H})$ y Egocentrismo ( $3 \mathrm{R}+2 /$ $\mathrm{R})$, se reclasificaron las respuestas como ausencia (0) y presencia (1).

Con estas recodificaciones se procedió a efectuar los análisis estadísticos no-paramétricos que implicaban el uso de la prueba de la mediana y las tabulaciones cruzadas, utilizándose el chi cuadrado y el coeficiente Fi como medida de asociación.

\section{Cuadro 1}

Resumen de los análisis de la prueba de la mediana de las variables estudiadas según rendimiento escolar

\begin{tabular}{|l|c|c|c|}
\hline \multicolumn{1}{|c|}{ Varrable } & Chr cuadrado & C.l. & ? \\
\hline Índice Ego & 5.40 & 1 & .02 \\
\hline Contenido Humano & 0.69 & 1 & .40 \\
\hline
\end{tabular}

En el Cuadro 1 se puede observar que en la prueba de la mediana existen diferencias estadísticas significativas sólo en el caso del Indice Ego, mientras que en la variable contenido Humano los resultdos indican que no existe diferencias entre lo sujetos de bajo y buen rendimiento escolar. 


\section{Cuadro 2}

Resumen de los análisis clit cuadrado de las variables estudiadas segín rendimiento escolar

\begin{tabular}{|l|c|c|c|}
\hline \multicolumn{1}{|c|}{ Varrable } & Chi cuacrado & G.I. & P. \\
\hline Respuesta de reflejo & 16.15 & 1 & .0001 \\
Movimiento humano & 2.29 & 3 & .51 \\
$\begin{array}{l}\text { Respuestad de } \\
\text { dimensionalidad }\end{array}$ & 0.94 & 1 & .33 \\
$\begin{array}{l}\text { Radiografiay } \\
\text { anatomia }\end{array}$ & 8.10 & 1 & .01 \\
$\begin{array}{l}\text { Afectos negativos y } \\
\text { contenidos depresivos }\end{array}$ & 0.11 & 3 & .74 \\
\hline
\end{tabular}

En lo que respecta al análisis realizado con el chi cuadrado, en el Cuadro 2, se puede observar que sólo existen diferencias estadísticamente significativas en las variables de respuesta de reflejo y de radiografía y anatomía, mientras que no hay diferencias en: movimiento humano, respuestas de dimensionalidad y afectos negativos con contenidos depresivos.

En el Cuadro 3 se incluye el análisis de los puntajes alcanzados en las respuestas del índice ego según el nivel de rendimiento escolar de los alumnos contrastados con la prueba de la mediana. Los resultados muestran que existen diferencias significativas entre ambos grupos, apreciándose que el grupo de alumnos de bajo rendimiento tienen la mayor proporción de casos con puntajes superiores a la mediana, lo que indicaría que estos alumnos estarían más centrados en si mismo, lo que estaría relacionado con una baja autoestima. 
Evaluación de la Autoestima en adolescentes con bajo rendimiento escolar

\section{Cuadro 3}

Prueba de la mediana del indice egocentrismo seguin rendimiento escolar

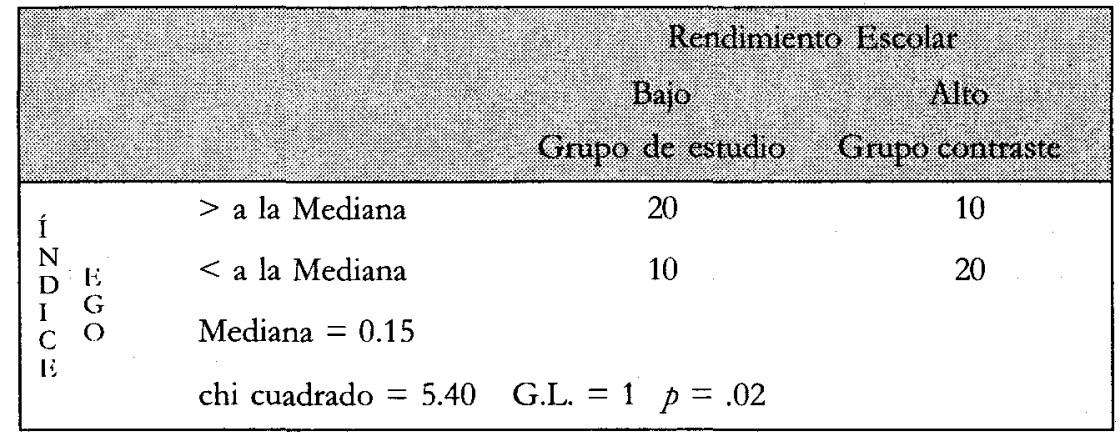

En lo que respecta a los resultados de la respueta de reflejo (ver Cuadro 4) notamos que existen diferencias significativas en la presencia y ausencia de las respuesta de reflejo según el nivel de rendimiento de los alumnos, pues a una chi-cuadrado igual a 16.15 le corresponde una probabilidad asociada igual a .00002 , obteniéndose así mismo un coeficiente de asociación Fi igual a .55. Además se debe notar que los sujetos de bajo rendimiento tienden a presentar una mayor proporción de respuestas de reflejo, lo que indica que estos alumnos en términos de narcisismo vienen desarrollando un mayor esfuerzo para poder desenvolverse y que tienen dificultades para la participación grupal, lo que estaría en relación a una baja autoestima.

En lo que se refiere a las respuestas de anatomía y radiografia, en el Cuadro 5 encontramos que si existen diferencias significativas según el nivel de rendimiento de los alumnos, alcanzándose un coeficiente de asociación $F i$ igual a .40 , encontrándose que lo sujetos de bajo rendimiento tienden a presentar una mayor proporción de respuestas de anatomía y radiografía, lo que nos indica el grado de preocupación por si mismo que estarian presentando y que se sienten aislados de interacciones significativas con el entorno, lo que correspondería a una inadecuada autoestima. 


\section{Cuadro 4}

Test chi cuadrado de la respuesta de reflejo seguin rendimiento escolar

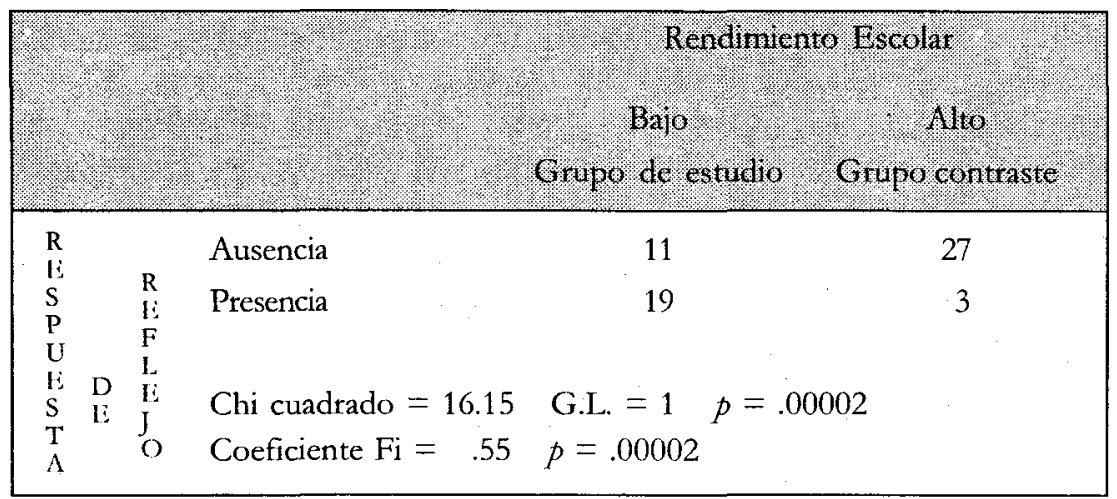

\section{Cuadro 5}

Test chi cuadrado de anatomia y radiografia según rendimiento escolar

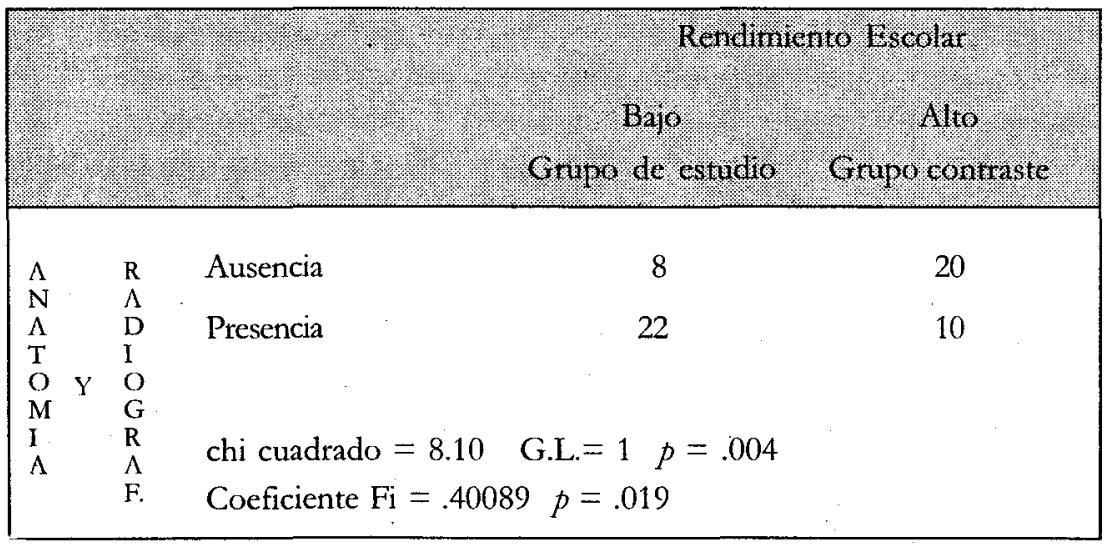


Evaluación de la Autoestima en adolescentes con bajo rendimiento escolar

\section{Discusión}

El Sistema Comprensivo de Exner, base de nuestro estudio enfatiza los aspectos estructurales de la técnica e incluye los últimos adelantos de la estadística y de la computación para un enfoque cuantitativo de los datos; pero no es nuestro único marco referencial. Por otro lado, las escuelas europeas de Rorschach, representadas especialmente por Nina Rausch de Traunbenberg (1993), también se encuentran dentro de una línea de trabajo que va de la interpretación cuantitativa a la cualitativa. Señala que si bien es importante la lectura de los datos cuantitativos, utilizando métodos estadísticos adecuados, cuando se trata de un número limitado de sujetos, se debe realizar un análisis profundo de los componentes de los diferentes factores; es decir, la exploración de las diversas dimensiones psicológicas en el Rorschach.

Nosotros nos hemos centrado en esta investigación sólo en aquellos elementos que en función de su especificidad estructural, podrían enfocarse sobre las dimensiones estudiadas, como es la autoestima en adolescentes, con niveles intelectuales promedio o superior al promedio y que presentaban bajo rendimiento escolar.

Hemos encontrado en nuestro medio estudios en adolescentes con bajo rendimiento escolar como son los de Orihuela (1974) y Zuñiga (1990). Y en cuanto a investigaciones en relación a nuestro tema de estudio, utilizando la Técnica del Psicodiagnóstico de Rorschach según Exner, realizadas en el extranjero, estaría la de Roderic (1991). Pero trabajos que relacionen ambos temas son muy poco frecuentes.

Nuestro estudio es el primer trabajo peruano en investigar estos puntos, que consideramos son básicos para el estudio evolutivo de la adolescencia. $Y$ si tomamos en cuenta que más del $48.1 \%$ de la población peruana está ubicada por debajo de los 19 años, podemos inferir la importancia de estudios en este grupo. (Instituto Nacional de Estadística e Informática, 1994). 
La muestra que hemos trabajado tiene características especiales dado que los sujetos presentan niveles de inteligencia normal o superior al promedio y sin problemas específicos de aprendizaje, lo cual nos lleva a pensar que podrá existir otro factor de indole psicológico que limita el rendimiento. La autoestima por ser un punto clave del proceso de desarrollo, podrá ser una de las causas interferentes en su aprendizaje escolar.

La autoestima domina la vida subjetiva del individuo determinando en gran medida sus pensamientos, sus sentimientos y su conducta. A primera vista parece tratarse de un fenómeno exclusivamente individual, personal o idiosincrático. Sin embargo, el autorretrato del individuo no constituye una obra estrictamente subjetiva, sino más bien, un retrato más o menos claro basado en su experiencia social.

En base a estas consideraciones pasaremos a discutir nuestros hallazgos comparando si existen diferencias en el nivel de la autoestima que presentan los adolescentes de 13 a 16 años de ambos sexos, con nivel intelectual normal o superior a lo normal y que presentan bajo rendimiento escolar; en comparación con los que tienen un rendimiento escolar superior al promedio. El trabajo nos ha permitido evaluar empíricamente la relación que existe entre lo intelectual y lo afectivo, que ha sido intuido pero pocas veces comprobado.

En el análisis de los resultados, encontramos que existen diferencias significativas en la presencia y ausencia de respuestas de reflejo (Fr), según el nivel de rendimiento de los alumnos. Podemos apreciar que los sujetos del grupo de bajo rendimiento tienden a presentar mayor proporción de respuestas de reflejo, lo que revela que en términos de narcicismo estos adolescentes vienen desarrollando un mayor esfuerzo para poder desenvolverse y que tienen dificultades para la participación grupal, lo que estaría en relación a la autoestima. Y que no es verdad que el adolescente tiene que ser tan narcisista para tener un adecuado rendimiento, ya que en el grupo de alto rendimiento, los resultados señalan que en este grupo el narcicismo se organiza como algo valioso. La autoestima les permite estar 
Evaluación de la Autoestima en adolescentes con bajo rendimiento escolar

a gusto y no enfatizar otros signos como el egocentrismo para llevar a cabo el cambio dentro de su proceso evolutivo.

La realidad demuestra que en el grupo de bajo rendimiento la presencia del signo $\mathrm{Fr}$ es una necesidad de mayor energía para compensar, que no necesitan los de alto rendimiento. El centrarse en sí mismo limita la energía para la tarea intelectual. Los de bajo rendimiento están más inseguros, más preocupados por los cambios que se dan en ellos, y todo eso lo expresan en su rendimiento, como una llamada de atención al medio.

Los resultados permiten afirmar que no existen diferencias entre los niveles alcanzados en ambos grupos de estudio en las respuestas de movimiento humano $\mathrm{M}$, lo que indicaría que en términos de mundo interno ambos grupos tienen similar capacidad para ubicarse en la realidad, controlar sus deseos, impulsos y fantasías. Indica además la existencia de recursos internos organizados y acequibles, que pueden posibilitar su buena adaptación al mundo exterior. Lo que nos lleva a plantearnos qué es lo que ocurre en la relación con los profesores, y con los padres del grupo de bajo rendimiento, ya que los dos grupos son similares estructuralmente y que es en los aspectos especificos donde aparecen las diferencias, las cuales vendrían desde fuera.

Las respuestas de reflejo son las que estarían dando las diferencias, con mayor o menor egocentrismo dentro del nivel del proceso evolutivo esperado.

Es importante destacar que en lo referente a las respuestas de anatomía y radiografía, (An+Xy) sí existen diferencias significativas, ya que los sujetos de bajo rendimiento tienden a presentar una mayor proporción de dichas respuestas. Esto nos indica el grado de preocupación por sí mismos que están presentando y que se sienten aislados de interacciones significativas con el entorno; además de ser una forma dolorosa de preocupación en la que se intenta resistir a la tensión e inhibir sus respuestas ante dicha situación. Es un intento más teórico que práctico para acercarse a las personas, con presencia de sentimientos de vacio, pesimismo, 
egocentrismo, agresión encubierta, temor a perder el control, represión, inhibición, ansiedad y rigidez en sus relaciones interpersonales.

Llama la atención que no son los contenidos depresivos con afectos negativos, los signos que establezcan diferencias en ambos grupos. Esto nos lleva a afirmar que las conductas depresivas serían la consecuencia y no la causa del bajo rendimiento.

El que no existan diferencias significativas en ambos grupos en los análisis de los puntajes obtenidos en las respuestas de movimiento humano $\mathrm{M}$ y contenido humano $\mathrm{H}$, explicaría que desde el punto de vista clínico no hay patología en el proceso de desarrollo de los sujetos en estudio. Los elementos valiosos de fantasía y potencial, creatividad se presentan en ambos grupos.

Al analizar el Indice de Egocentrismo, observamos que sí existen diferencias significativas en ambos grupos y que el grupo de bajo rendimiento tiene la mayor proporción de casos. Es interesante resaltar que el egocentrismo es una característica natural de la persona que probablemente funciona como un factor positivo siempre que no sea "excesivo" o "insuficiente". Nuestros hallazgos señalarian que el grupo de bajo rendimiento estaría más centrado en sí mismo, lo que estaría relacionado con una baja autoestima. Es decir, a una tendencia "autoenfocante" que puede set concebida como una defensa o un ocultamiento de sentimientos más profundos y de poco mérito personal (Rovira, 1982). El egocentrismo en el grupo de bajo rendimiento resulta una carga muy difícil de manejar, constituyendo el centro de su vida ideacional. $Y$ en este caso los problemas de autoestima disminuyen el aprendizaje distanciándoles de la realidad.

Al utilizar el Psicodiagnóstico de Rorschach para estudiar el proceso adolescente, se puede evaluar lo que está sucediendo desde una perspectiva más allá de los fenómenos externos, los que sólo lo presentan como un problema más severo, y no como un aspecto esencial del proceso adolescente. Se puede llegar a aproximaciones más precisas, evitando caer en 
la percepción de una patología inexistente y sí enfatizar cómo determinadas conductas son el inicio del cambio. La autoestima es crucial en la adolescencia, es lo que permite establecer las diferencias en los dos grupos estudiados, ya que estando conformados por adolescentes igualmente inteligentes puede explicar las diferencias de rendimiento.

Hemos podido comprobar que la inteligencia es adecuada, por lo que utilizar refuerzos educacionales no sería tan necesario como utilizar los refuerzos emocionales, ya que no existen dificultades ni de funciones ni de cociente intelectual. Es necesario fortalecer el programa para trabajar aspectos emocionales tanto con los profesores, como con los padres y con el grupo de referencia.

Esta investigación nos ha permitido comprobar la relación que existe entre la teoría y la realidad. Al conocer los cambios naturales que ocurren durante la adolescencia es posible aproximarse a ella desde la óptica de la normalidad y apreciar que los problemas que ocurren pueden ser más de índole coyuntural que estructural y que por lo tanto no es indispensable pensar en la patología al estudiar al adolescente.

La técnica utilizada a nuestro juicio es adecuada, porque supera la utilidad tanto de los cuestionarios de personalidad como de las pruebas de inteligencia, cuando se trata de individuos inteligentes y de bajo rendimiento escolar.

Finalmente podemos indicar que es nuestra intención que este trabajo llegue a constituir un modelo de investigación, el cual nos facilite el estudio desde una perspectiva holística a través del Psicodiagnóstico de Rorschach con el Sistema Comprensivo de Exner, de los diferentes constructos implicados en las diversas etapas del desarrollo evolutivo. Esto nos permitirá llegar a conocer cada vez más nuestra realidad, por medio de investigaciones que tengan un valor práctico y que constituyan un aporte al quehacer diario de los psicólogos en el Perú. 


\section{Conclusiones}

- El análisis psicométrico de los intrumentos utilizados, el Psicodiagnóstico de Rorschach según el Sistema Comprensivo de Exner y el WISC-R, nos confirma que son pruebas confiables y adecuadas para el desarrollo del estudio.

- El Sistema Comprensivo del Pșicodiagnóstico de Rorschach según Exner, ha demostrado ser un buen instrumento para evaluar la autoestima de los adolescentes.

- Los sujetos del grupo de bajo rendimiento presentan una baja autoestima en relación al grupo de alto rendimiento, lo cual es resultado de sus déficits afectivos.

- Los sujetos de los grupos de alto y bajo rendimiento, tienen una capacidad similar para ubicarse en la realidad, controlar sus deseos, impulsos y fantasías.

- Los resultados son válidos para la muestra y sugieren hipótesis de trabajo con estos mismos signos en una muestra más amplia que incluya adolescentes de diferentes niveles socio económicos.

\section{Referencias}

Alcock, T. (1975). La prueba del Rorschach en la práctica. México: Fondo de Cultura Económica.

Andronikof- Sanglade, A. (1993) L'abstraction au Rorschach comme mécanisme d'anti-symbolisation. Bulletin Societé $d u$ Rorschacb et des Méthodes Projectives de Langue Francaise, (37) p. 71- 91.

Caplan, G. (1983). Psicología Social de la Adolescencia. Buenos Aires: Editorial Paidos.

Chabert, C. (1991). Narcissime et rélations d'object à l'adolescence: apports des épreuves proyectives. L'Enciclopédie Chirurgical Psychiatrie. 3721 B B10.

Exner, J. E. Jr. (1992). A Rorschach Workbook for the Comprensive System. Anheville: North Carolina. 
Evaluación de la Autoestima en adolescentes con bajo rendimiento escolar

Exner, J.E. Jr. (1993). The Rorschach Comprensive System. Vol. 1 (3rd. cd.). Nueva York: Wiley.

Freud, S. (1972). Obras Completas. Tomo I. Biblioteca Nueva Visión Madrid Garrido Lecca, M. (1995). Evaluación de la autoestima en adolescentes con bajo rendimiento escolar a través del Psicodiagnóstico de Rorschach según Exiner. Tesis de Magister en Psicología, PUCP.

Guilford, J.P. (1954). Psychometrics Methods. New York, Mc Graw Hill. Halpern, F. (1953). A Clinical Approach to Childern's Rorschach. New York: Grone Estratton.

Instituto Nacional de Estadística e Informática . (1994). Perú: Resultados Definitivos. Perfil Socio-Demográfico. Lima, Perú: INEI

Jeammet, P. (1993). Les test projectifs à l'adolescence. Bulletin Societé du Rorschach et des Métbodes Projectives de Langue Francaise. (37), 57-69.

Marcelli, D. (1986). Psychopatologie de l'adolescent. Paris: Masson.

Orihuela Santolaya, César (1974). Los hábitos inadecuados de estudio como factor de bajo rendimiento, sus medidas correctivas $y$ análisis de otros factores de rendimiento escolar. Tesis, U.N.M.S.M.

Piaget, J. (1971). Los estudios del desarrollo intelectual del niño y del adolescente. Buenos Aires: Nueva Visión.

Rausch De Traubenberg, N. (1993). Le Rorschach à l'adolescence: la clinique du normal. Bulletin Societé du Rorschach et des Méthodes Projectives de Langue Francaise. 37, 7- 39.

Roderic, L. (1991) The egocentricity index as ameasure of self-esteen and egocentric personality style for inpatient adolescents. Perceptual and Motor Skills, 73, 907-914.

Rosenberg, M (1983). La autoestima del adolescente y la sociedad. Buenos Aires: Paidós.

Rovira, F. (1983). El Rorschach Nuevas Aportaciones de J.E. Exner al Sistema Comprensivo. Madrid: Siglo Ventiuno de España. Editores, S.A.

Wechsler, D. (1974). Wechsler Intelligence Scale for Cbildren Revised. Nueva York: The Psychological Corporation.

Zuñiga Majuan, Rosa Elvira (1990). Estudio del ajuste de la personalidad de un grupo de adolescentes varones con problemas de conducta y/o bajo redimiento escolar. Tesis, U.S.M.P. 